\section{Failure Detection}

In spite of preflight test concerns, pilots readily detected all failures introduced during the flight tests with the sole exception of ADF failures, which resulted in frozen indicators, both head-up and head-down. This is a criticism of the ADF design, not the HUD.

\section{Unusual Attitude Recoveries}

Initial unusual attitude recoveries were performed with navigation data removed from the HUD. The HUD ratings were generally threes. During FAA flights, the pilots flew the unusual attitudes with navigation data shown. The added clutter and conflicting rotational cues caused the ratings to deteriorate to the seven or eight level. As a result, the HUD was modified to provide for automatic declutter during unusual attitudes. A yoke-mounted declutter button was also added.

\section{ILS Symbology}

Maintaining geographical orientation appears to be more difficult with a HUD than with a head-down display. The ILS symbology used a small cross to show raw deviation and a single cue airplane to show flight director commands. Some pilots reported difficulty with the raw data symbol, confusing it with split cue flight director needles.

\section{Choice of Pilots}

The combination of test pilots and several operational pilots worked well. Generally, the two groups agreed on relative ratings. However, operational pilots tended to rate the display readability and handling one or two rating points better than the test pilots.

\section{Lessons Learned}

\section{Use of Surrogate Aircraft}

The single-engine Grumman allowed an inexpensive screening tool to develop HUD symbology.

\section{Effect of Workload}

At the same time, care must be taken when using a surrogate aircraft. We recognized that initial King Air flights would need to check the dynamic response of the HUD to ensure satisfactory handling using the HUD, but did not recognize that the increased workload would be so dramatic for HUD functions such as using the checklist.

The effect of workload caused by high-density air traffic was not anticipated. Future display evaluations should ensure that representative ATC workload is present and not perform evaluations in a sterile environment.

\section{Test Pilots vs Operational Pilots}

The combination of two test pilots with two to four operational pilots seems to give a suitable combination of critical evaluation and a variety of pilot background.

\section{Unusual Attitudes}

The original flight tests tasked the pilots with recovering from unusual attitudes with decluttered HUDs. When the test was repeated with navigation data shown, the HUDs were significantly downrated. Future HUD flight tests should conduct unusual attitude recoveries with maximum symbol density (i.e., minimum declutter).

\section{References}

${ }^{1}$ Anon., "Transport Category Airplane Electronic Display Systems," Federal Aviation Administration AC-25-11, July 1987.

${ }^{2}$ Anon., "Equipment, Systems, and Installations," Airworthiness Standards: Normal, Utility, Acrobatic, and Commuter Category Airplanes, 14 CFR Pt. 23, Jan. 1994, Paragraph 23.1309.

${ }^{3}$ Anon., "Equipment, Systems, and Installations," Airworthiness
Standards: Transport Category Airplanes, 14 CFR Pt. 25, Jan. 1994, Paragraph 25.1309.

${ }^{4}$ Monagan, S. J., and Smith, R. E., "Head-Up Display Flight Tests," Proceedings of the 24th Symposium, Society of Experimental Test Pilots (Beverly Hills, CA), Society of Experimental Test Pilots, Lancaster, CA, 1980, pp. 75-87.

${ }^{5}$ Haworth, L. A., and Newman, R. L., Test Techniques for Evaluating Flight Displays, NASA TM-103947, Feb. 1993.

${ }^{6}$ Anon., Flight Test Guide for Certification of Normal, Utility, and Acrobatic Category Airplanes, Federal Aviation Administration AC23-8, Oct. 1987.

\section{Integrated Digital Avionics to Improve Aircraft Environmental Control Systems}

\author{
Kenneth P. Katz* \\ University of Michigan, \\ Ann Arbor, Michigan 48109-2125 \\ and \\ James L. Ozimek $\dagger$ \\ Boeing Defense and Space Group, \\ Philadelphia, Pennsylvania 19142-0858
}

\section{Introduction}

TNEGRATED digital avionics technology has been an innovation of tremendous importance to aircraft design and operations. The primary application of avionics traditionally has been in the areas of missions systems, crew stations, and flight controls. More recently, aircraft utilities including environmental control, fuel, hydraulics, and electrical power have also been interfaced with digital avionics.

There are several benefits that result from interfacing these utility systems with a digital avionics system.

1) Dedicated controls and displays can be replaced by multifunction controls and displays. The elimination of these dedicated components decreases weight and costs and increases reliability.

2) Automating the routine operation of utility systems improves operational effectiveness and safety by lowering operator workload.

3) Built-in test (BIT) and failure recording simplify maintenance.

4) Systems that are digitally interfaced can share data. Through the use of this shared data redundant measurements can be reduced with consequent decreases in weight and costs.

5) Mission reliability can be increased by dynamic reconfigurations that retain mission capabilities, even with hardware failures.

The V-22 Osprey is a tiltrotor aircraft being developed by the team of Bell Helicopter Textron and The Boeing Co. It features a digital avionics architecture that integrates all of the systems of the vehicle. The environmental control system (ECS) of the V-22 provides several examples of the benefits of integrating utility systems with digital avionics.

\footnotetext{
Received Oct. 22, 1994; accepted for publication Aug. 11, 1995 Copyright (C) 1995 by the American Institute of Aeronautics and Astronautics, Inc. All rights reserved.

*Technical Specialist, Mechanical Design; currently Graduate Student, Program in Manufacturing. Senior Member AIAA.

†Senior Technical Specialist, Mechanical Design, Helicopters Division, P.O. Box 16858, M/S P24-62.
} 


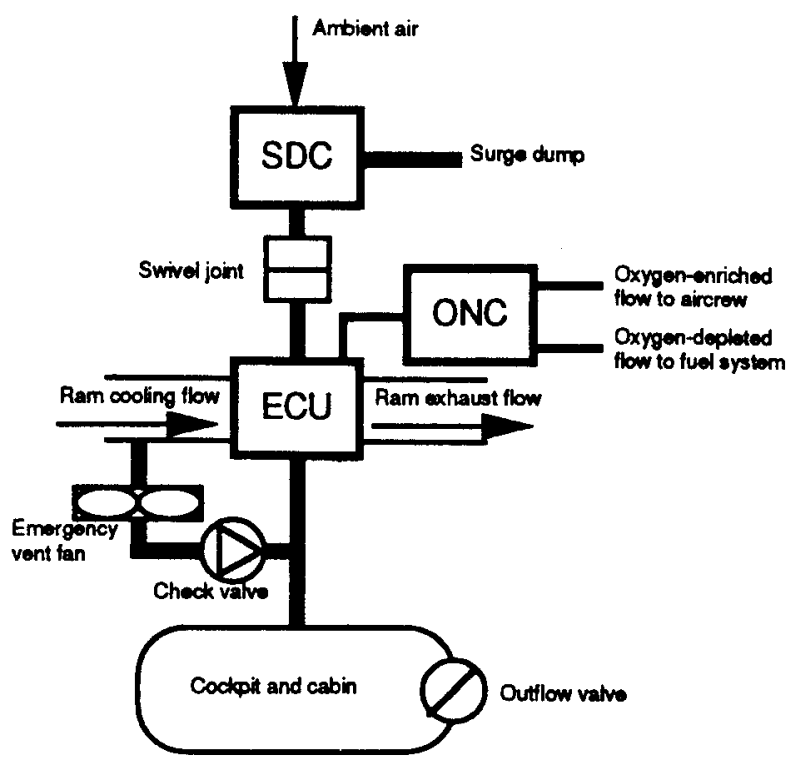

Fig. 1 ECS (AFACS, sensors, and ECSC omitted for clarity).

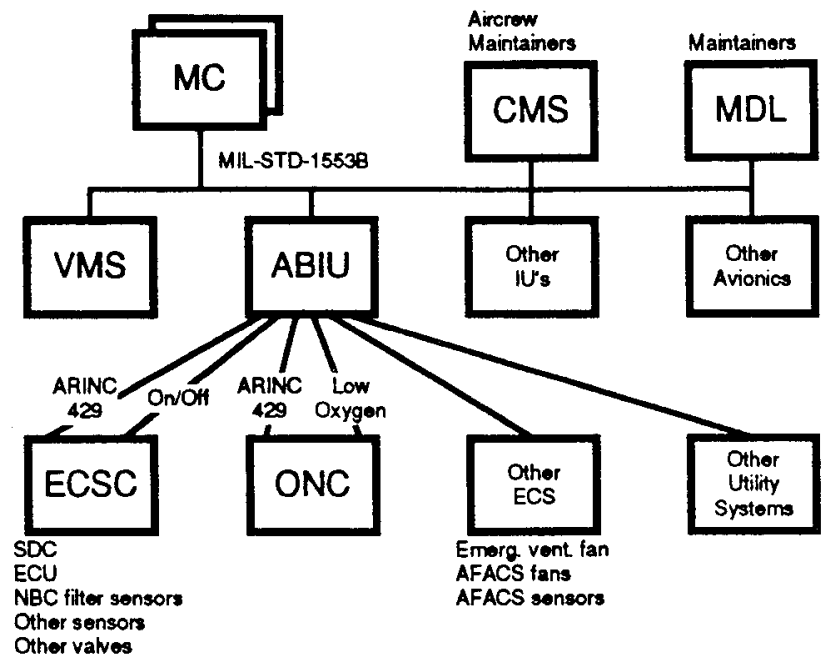

Fig. 2 IAS with emphasis on ECS interfaces.

\section{System Description}

Three subsystems comprise the V-22 ECS (Fig. 1).

1) A shaft-driven compressor (SDC) is powered by a midwing gearbox and supplies compressed air to the ECS. The environmental control unit (ECU) heats or cools, dehumidifies, and filters the compressed air from the SDC. The ECU consists of an air cycle machine, heat exchangers, and optional nuclear, biological, and chemical (NBC) filter. Distribution ducts, valves, and sensors control the flow of conditioned air to create a habitable cockpit and cabin environment that can be pressurized to protect the crew and passengers against NBC agents. The SDC, ECU, and related components are controlled and monitored by an ECS controller (ECSC). An emergency ventilation fan can be used to bypass the SDC and ECU.

2) Part of the output of the ECU is routed to an oxygennitrogen concentrator (ONC), which forms the core of the onboard inert gas generating system (OBIGGS) and on-board oxygen generating system (OBOGS). The ONC uses molecular sieves to create an oxygen-enriched air supply for aircrew breathing through oxygen masks and an oxygen-depleted air supply to inert the ullage in the fuel cells for ballistic protection.

3) The avionics forced air cooling system (AFACS) intakes ambient air and removes particulates with centrifugal filters.
The air is then collected in a plenum and passed through an NBC filter/coalescer before being passed through two avionics racks and the nose avionics bay. Two intake fans and two exhaust fans force the air through the avionics bays. Pressure and temperature switches sense AFACS failures.

Each subsystem of the ECS interfaces with the integrated avionics system (IAS) (Fig. 2). The IAS is based on the MILSTD-1553B bus architecture. A mission computer (MC) is the bus controller and executes central processing with another MC acting as a spare. Remote terminals on the redundant MILSTD-1553B buses include the vehicle management system (VMS) for flight control, cockpit management system (CMS) for control and displays, mission equipment for navigation and other tasks, and four interface units (IU). The IUs multiplex and demultiplex various analog, discrete, and ARINC 429 serial digital signals to interface them with the MIL-STD-1553B buses. The avionics bay interface unit (ABIU) is the IU that interfaces with the ECS. Aircrew and maintainers operate and monitor the ECS through the CMS. Failures detected by BIT are stored in the MC memory. After the completion of a flight maintainers download the fault data in MC memory with the mission data loader (MDL).

\section{Features of ECS Avionics Interface}

Various features of the V-22 ECS and its IAS interface beyond the basic control and monitoring functions illustrate the benefits of applying digital avionics to utility systems.

The utility hydraulic system pump is driven by the same gearbox that turns the SDC. During engine starts, the power demands of the hydraulic engine start motors require the SDC load to be removed from the gearbox. This is automatically accomplished by the IAS. The VMS transmits to the MC that an engine start is in progress. By means of the IU, the MC commands the ECSC to de-energize the control solenoid that opens the guide vanes on the inlet of the SDC. When the solenoid is de-energized SDC ceases to produce compressed air.

Since the V-22 wing must rotate 90 deg to its stowed position to permit the aircraft to fit in the hangar deck of ships, the compressed air from the SDC must pass through a swivel joint to reach the remainder of the ECS, which is located in the fuselage. Rotating the wing while the swivel joint is pressurized would shorten the life of the joint. When the operator executes a wing stow or unstow, the SDC is automatically unloaded by IAS command to extend swivel joint life.

The emergency ventilation fan can provide a flow of fresh air to the cockpit and cabin when the SDC and ECU are not operating. However, the emergency ventilation fan bypasses the NBC filter. If the fan were used in a contaminated environment, the cockpit and cabin then would be contaminated. The ECSC senses the presence or absence of the ECU NBC filter and transmits this data to the IAS. The IAS inhibits the operator from activating the emergency ventilation fan when the NBC filter is installed, although this inhibition can be overridden. This feature reduces the probability of an operator error that would imperil the crew and passengers.

The diagnostic capabilities of BIT significantly reduce maintenance requirements by assisting troubleshooting and minimizing maintenance-induced failures. BIT not only ensures that full system operational capabilities are maintained, it also provides the information required to reconfigure the system for mission accomplishment in spite of failures. The ECSC and ONC have internal BIT capabilities, whereas AFACS uses the $\mathrm{MC}$ to execute its BIT logic. Each subsystem has a nonintrusive periodic BIT mode that senses failures during normal operations. The ECSC and ONC also have intrusive initiated BIT (IBIT) modes to comprehensively determine their readiness for flight. While IBIT is in progress, it halts normal system operation and exercises all of the components of the subsystem. When conducting IBIT, the ECS ceases to supply conditioned 
air. This makes it undesirable to conduct ECS IBIT while airborne. The VMS receives signals from switches on the landing gear to determine if the vehicle is flying. This information is transmitted to the IAS and used for many purposes, including inhibiting ECS IBIT while airborne.

The means by which the CMS informs the pilots of ECS failures must be carefully designed to convey the information that they require to make operational decisions without excessive complication. This human interface design philosophy is known as the functional failure approach, in contrast to the hardware failure method that annunciates the failure of specific components and then forces the operator to evaluate the effects and operational implications of the failure. For example, the CMS would annunciate outflow valve failure using the hardware failure method if the ECSC determined that the valve was not responsive to its commands. In contrast, CMS would send an ECS NBC failure message to the pilot in the event of an outflow valve failure, since this valve is required for the compartment overpressurization that is part of the NBC protection scheme.

For fault isolation purposes, the ONC must be informed that it is getting an adequate supply of compressed air from the ECS to detect and isolate internal failures with BIT. The ECSC uses an SDC discharge pressure sensor to determine if the compressed air supply from the ECU is adequate and then transmits this information to IAS. The IAS then supplies this information to the $\mathrm{ONC}$, eliminating the need for a dedicated $\mathrm{ONC}$ inlet pressure sensor.

On the ground, in hover, and at low forward speeds, the avionics cooling airflow is provided by the four AFACS fans. At high forward speeds, the dynamic pressure at the inlets of the AFACS particle separators alone is sufficient to cool the avionics. Continuing to operate the fans in this part of the flight envelope would result in excessively high stagnation temperatures in the flow. The VMS transmits the current airspeed to the IAS and the MC uses this data to automatically command the AFACS fans on and off through the IU. When the fans are off, the cooling air passes through bypass ducts. The MC will command the AFACS fans to run at any airspeed if temperature switches in the avionics bays detect an overtemperature condition. In the absence of an avionics interface, either the ECS would need a separate airspeed sensor for the ECS or else pilot workload would increase.

Two other examples also illustrate the use of avionics data in lieu of dedicated sensors or wiring. The IAS transmits outside air temperature data from the VMS to the ECSC via the IU and MC. This information is used to predict compressed air temperatures in the ECU and prevent the exposure of ECS components to excessive temperatures. Its availability on the MIL-STD-1553B obviates the need for a dedicated ECS outside air temperature sensor. Similarly, the IAS transmits ambient static air pressure to the ECSC. In combination with data from the SDC discharge pressure sensor, the ECSC uses the static air pressure data to monitor SDC performance.

The IAS monitors temperature switches in the AFACS fan motors and automatically shuts the fans off if they overheat. The IAS also monitors temperature switches in the avionics bays that sense bay overheating. Since the value of the avionics greatly exceeds that of the fans, in the event of avionics bay overheating, the MC will turn the AFACS fans on, even if their motors are overheated.

\section{Summary}

The V-22 ECS illustrates the variety of improvements to operational effectiveness and maintainability that result from integrating utility systems and digital avionics. 